\title{
Comparison of estimates calculated on the energy flow problem basis and using testing equations method
}

\author{
Elena Kochneva ${ }^{1}$ and Aleksandar Sukalo ${ }^{2}$ \\ ${ }^{1}$ Automated Electrical Systems Department, Ural Federal University named after the first President of Russia B.N. Yeltsin, 19, Mira St., \\ Yekaterinburg, Russia \\ ${ }^{2}$ Elektroperenos - Elektroprijenos BiH, Banja Luka, Bosna and Hercegovina
}

\begin{abstract}
The article is devoted to the electrical energy measurements estimates calculation. Automatic Meter Reading systems are the source of measurements. Two methods are compared. The first one is based on the energy flow problem, which allows to receive the estimates of the electrical energy flows and losses on all elements of the electrical network. Calculations using the first method were made using the software product "Balance". The second technique is simplified and uses a system of testing equations to calculate the measurements estimates. Comparison showed the use of a simplified procedure gives adequate accuracy on condition of sufficient redundancy level.
\end{abstract}

\section{Introduction}

The current state of the electric power industry is characterized by the following problems [1]:

- a high level of commercial losses,

- the difficulty of determining the commercial measurement information reliability degree,

- the complexity of the element-wise calculation of electrical energy technical and commercial losses in confined networks.

Many enterprises, grid and generating companies are equipped by Automatic Meter Reading systems. These systems include the modern microprocessor devices that provide high accuracy measurements and time synchronization. The main purpose of these systems is to provide financial settlements for electricity with reliable and operative measuring information. At the same time, the level of commercial power losses remains high. Despite the technical complexity of modern accounting systems, mathematical processing of the data is practically not done.

Existing approaches to measurement data processing allow to solve a wide range of problems: identification of unreliable data, localization of sources of commercial power losses, assessment of the characteristics of electricity metering complexes, and others [2].

\section{Energy flow calculation on the network elements using energy flow problem}

The task of calculating energy flows and losses on all elements of the electrical network using measurement information was defined as the energy flow problem.
Initially, attempts to model power distribution modes were associated with the use of steady-state equations [35]. However, it was found out that averaging the modes over a time interval leads to the appearance of unrecoverable unbalances in steady-state equations. The main reason is topological changes in the network [6]. The energy flow problem state system differ from the traditional state estimation one [7-11]. The electric energy balances equations in the nodes and branches of the network are to be used as the state equations describing the process of energy distribution. These equations are identities for any topological and mode changes.

Since the measured values of electrical energy contain metrological errors, the balance energy ratios in the nodes and branches of the network diagram, which include these measurements, may be nonzero. The energy flow problem allows to get the estimated energy flows and the balance ratios for these calculated flows will be correct. Calculations are made on the basis of the energy flow problem mathematical model. It is important to note that when using measured energy flows, the balance equations will include technical and commercial energy losses, and when compiling balances for the calculation model, the equations will include only technical losses.

The objective function of the energy distribution problem is the weighted squares sum of relative measurement errors:

$$
F=\sum_{l=1}^{K} a_{l}\left(\frac{W_{l}^{\text {meas }}-W_{l}^{\text {calc }}(X)}{W_{l}^{\text {meas* }}}\right)^{2} \rightarrow \min
$$

where $K$ is the number of energy measurements for the power system fragment under consideration. 


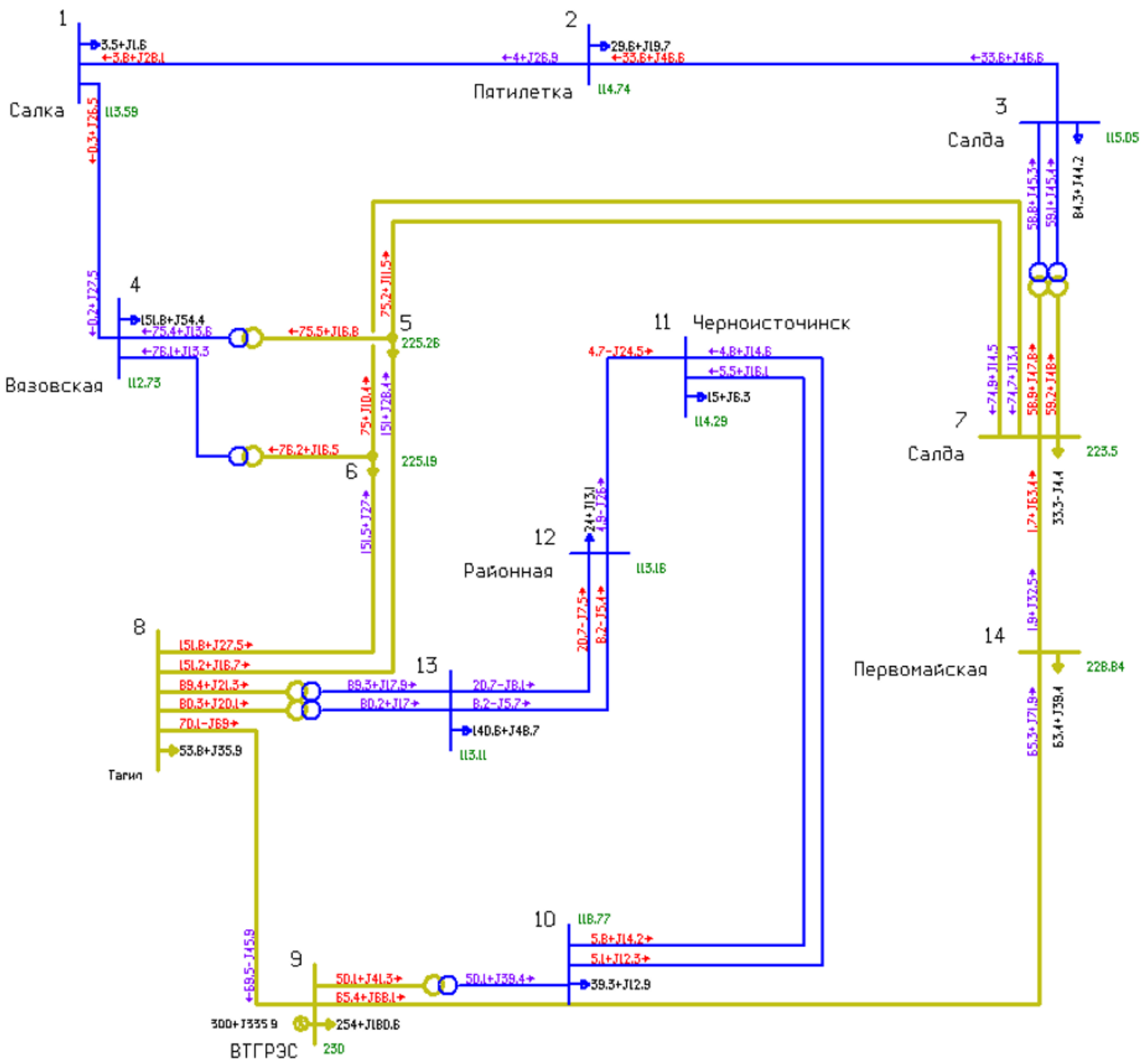

Fig 1. Test scheme

The weight coefficients $a_{l}$ from (1) are necessary to take into account the information on the relative errors of automatic meter reading complexes [12-13].

Thus, the network model for the solution of the energy flow problem is the traditional equivalent electric circuit with lumped parameters. It should be noted that the equivalent circuit parameters determine only the amount of active and reactive energy losses on the longitudinal and transverse elements of the network. The magnitude and direction of energy flows are determined by the available nodal and linear measurements, which are located in the branches and nodes of the equivalent circuit of the network under consideration.

\section{Testing equations method for energy flows calculated analogues estimation}

The algorithm for estimation the energy flows calculated analogues can be based on the testing equations system.

The method is based on the small coherence of the energy flow problem state equations. This method is an a priori one. According to the terminology of the state estimation theory, equations that include only the measured variables are called testing ones. The unbalance (mismatch) of such an equation will be zero if all the measurements in its composition contain no errors, and the unbalance is different from zero if the measurement includes an error. The presence of gross errors leads to the appearance of significant unbalances. Thus, the method of testing equations is close to the method of comparing actual and permissible imbalances, but it allows to analyze a connected balance equations system.

In real power systems, there is almost never a complete coverage of connections by energy measurements, in this case a testing equations system can be obtained from the energy flow problem state equations system by mathematical exclusion of all unmeasured variables.

The topological method of forming the testing equations system is visual and is based on the topology of the network and the placement of measuring complexes. The formalized procedure for obtaining control equations (the algebraic method) is based on the elimination of all non-measured variables from the energy flow problem state equations system by algebraic means. 


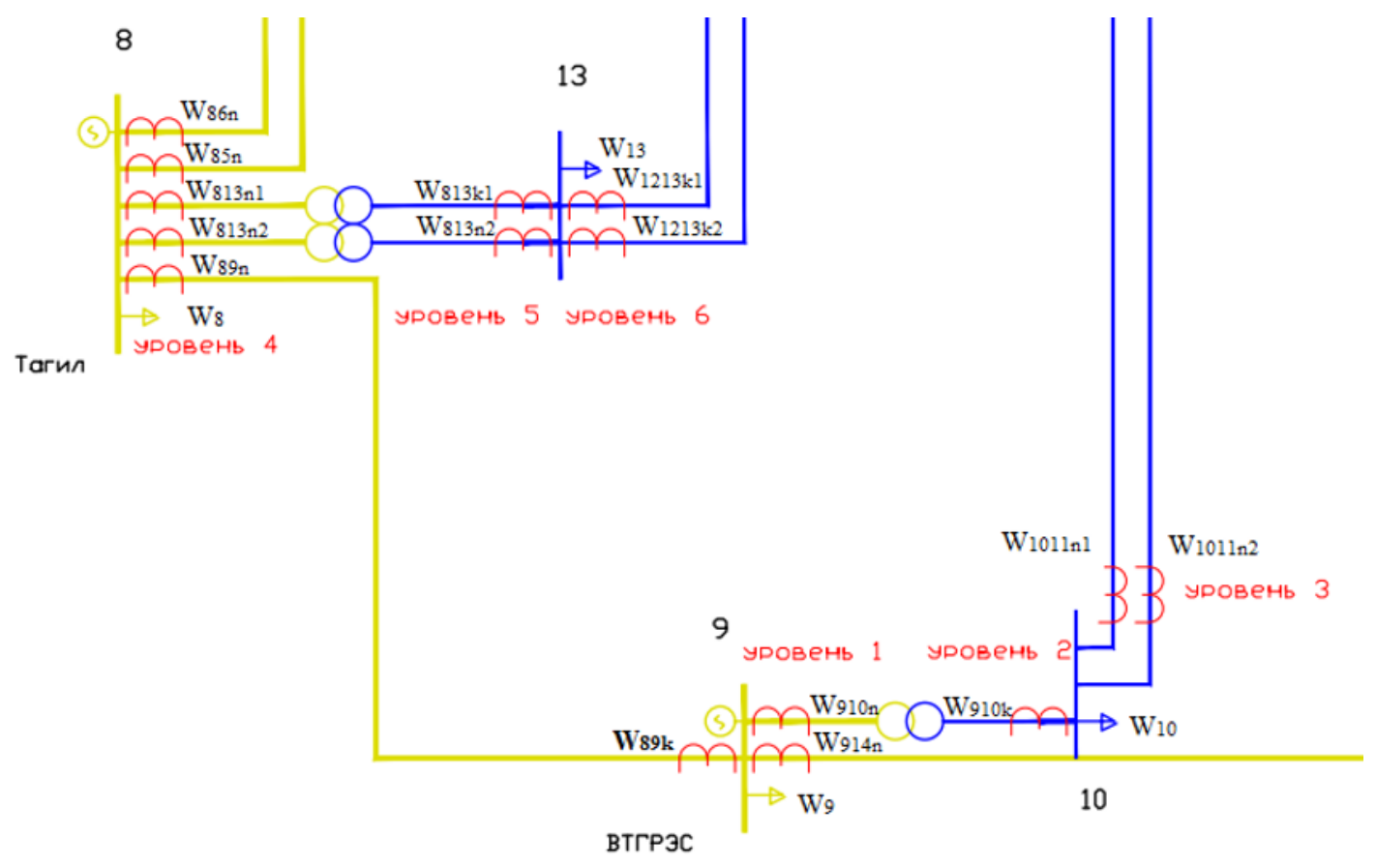

Fig 2. Line 8-9k measurement levels

Having transferred all the nodal and linear streams of energy to the left side, a system of linear equations with variables can be obtained. Considering nodal and linear energy flows as equal variables, all unmetered variables can be excluded by the Gauss method.

The presence of redundant measurements and the testing equations system construction makes the calculated measurement analogue computation possible. Each testing equation allows to compute the calculated measurement analogue. The final calculated measurement estimate can be obtained based on these calculated analogues. This process is described in [] in detail.

\section{Comparison of estimates calculated on the energy flow problem basis and using testing equations method}

To compare the proposed methods, a scheme of the real power system fragment was compiled. Figure 1 shows the circuit configuration under consideration.

The software complex "Balance" is intended for solving tasks on calculation, analysis and optimization of electrical networks modes, as well as systems of various configurations and arbitrary dimension. The energy flow problem for the network in question was solved using the software package "Balance". The results obtained during the calculation in the software package will be compared with the results of the manual calculation performed using the testing equation system.

Equivalent circuit including the load capacities of the nodes, as well as measuring of the electrical energy at all points where the Automatic Meter Reading complexes are installed are introduced into the program complex. The accuracy classes of the measuring complexes are also entered in the "Balance". The calculation result is the computed estimates of all electricity flows on network elements, as well as technical losses.

The lineal measurement $8-9 \mathrm{k}$ is selected as an investigated one. Figure 2 shows the levels of its measurement. That is, the nodal energy is measured not only by means of a specific Automatic Meter Reading complex installed on the outgoing line, but also it is reserved by a number of other measuring complexes located at a distance. In general, the composition of the measurements is redundant, which makes obtaining a testing equation system and computing a number of calculated analogues of measurement possible. The testing equations system is:

$$
\left\{\begin{array}{l}
W_{12 n}+W_{23 k}-W_{2}+d W_{23}+d W_{12}=0 \\
W_{14 k}-W_{12 n}-W_{1}+d W_{14}=0 \\
W_{45 n}+W_{45 k}+d W_{45}=0 \\
W_{46 n}+W_{46 k}+d W_{46}=0 \\
W_{86 n}-W_{46 k}-W_{6}+d W_{86}+W_{67 k}+d W_{67}=0 \\
\ldots
\end{array}\right.
$$

There are 27 redundant measurements in the network under consideration. The number of testing equations is the same. Testing equations system construction allows to solve a number of problems: estimate observability, to allocate the metering complexes [13-14], to reveal bad data [15].

In previous studies [15] it was shown that the use of more than 6 levels to calculate the estimated analogue does not lead to an increase in the accuracy of calculations. In this case, 6 measurement levels were accepted for calculations. First verifying equation is:

$$
W_{89 k}^{1}=-W_{910 n}-W_{914 n}-W_{9}
$$


The accuracy of the calculated estimate is $0.66 \%$ at a measurement complex accuracy of $1 \%$. Calculated value is $34,6679 \mathrm{kWh}$. The same variable calculated using Energy Flow Problem solving in «Balance» is 34,658 $\mathrm{kWh}$.

The final calculated analog is computed using the results of verifying equations taking into account the accuracy of each. The verifying equation accuracy is calculated through the accuracy classes of all Metering complexes whose indications are included in the verifying equation.

Accuracy calculation for the first verifying equation [16]:

$$
\delta_{1}=\sqrt{\left(\delta W_{910 n}\right)^{2}+\left(\delta W_{914 n}\right)^{2}+\left(\delta W_{9}\right)^{2}}
$$

Similar comparisons were made for a number of measurements. In all cases, the difference of the calculated estimates was less than $1 \%$, which is within the limits of the permissible errors of the Automatic Meter Reading complexes.

\section{Conclusion}

Obtaining the calculated measurement estimate allows to solve the problem of Automatic Meter Reading systems bad data validation. The ways of obtaining such estimates can be different. Studies and test calculations have shown that, if it is necessary to obtain a calculation estimate for single measurements, it is possible to use simplified methods based on a testing equations system. The magnitude of the calculated estimate is practically the same as the value obtained by solving the complete energy flow problem.

\section{References}

1. A. Pazderin, V. Samoylenko, Localization of nontechnical energy losses based on the energy flow problem solution. Proceedings of the 6th IASTED Asian Conference on Power and Energy Systems, AsiaPES 2013, pp.100-103, (2013).

2. A. Egorov, E. Kochneva, A. Pazderin, Detection of systematical errors of AMR system complexes, Advanced Materials Research 960-961, pp 1342-46, (2014).

3. A. Monticelli, F. F. Wu, M. Yen, Multiple Bad Data Identification for State Estimation by Combinatorial Optimization. Proceesings of the PICA Conference, pp. 452-460, (1985)

4. X. Nian-De, W. Shi-Ying, Y. Er-Keng, A new approach for Detection and Identification of Multiple Bad Data in Power System State Estimation. IEEE Transactions on Power Systems, PAS-101, no. 2, pp.454-462, (1982).

5. F. Schweppe, J. Douglas, D. Rom, Power System State Estimation, Part I - Exact Model, IEEE
Transactions on Power Apparatus and Systems, PAS-89, pp. 120-125, (1970).

6. A. Pazderin, S. Kokin, A. Egorov, E. Kochneva, Solution of energy flow problem using state estimation technique, ECON Proceedings (Industrial Electronics Conference), pp. 1736-41.

7. Abur A, Exposito A G, Power System State Estimation. Marcel Dekker inc., New York, (2004)

8. A. Gamm, I. Kolosok, Bad data detection in measurements in electric power system. Nauka, Novosibirsk, Sib. Enterpr. RAS, (in Russian), (2000).

9. F. Schweppe, J. Douglas, D Rom, Power System State Estimation, Part II - Approximate Model, IEEE Transactions on Power Apparatus and Systems, PAS-89, pp. 125-130, (1970).

10. A. Monticelli, Electric Power Syaytem State Estimation. Proceedings of the IEEE, vol. 88, no.2, pp. 262-282, (February 2000).

11. P. Chusovitin, I. Polyakov, A. Pazderin, Threephase state estimation model for distribution grids. Proceedings of International Conference on the Science of Electrical Engineering ICSEE, (2016).

12. E. Plesniaev, A. Pazderin, Data acquisition system faults detection. Proceedings of IEEE Conference on Contril Applications, pp.1390-94, (2003).

13. E. Plesniaev, A. Pazderin, Analysis of the computation techniques for energy flow problem solving. Proceedings of the International Conference on Computer as a Tool II EUROPCON, pp.1469-1472, (2005).

14. A. Pazderin, A. Egorov, S. Eroshenko. The energy meters allocation in electric systems on the basis of observability theory, Proceedings of 9th Conference on Environment and Electrical Engineering, EEEIC, pp. 167-170, (2010).

15. A. Pazderin, E. Kochneva, Bad data validation on the basis of a posteriori analysis. Proceedings of the IEEE International Energy Conference ENERGYCON, pp. 386-391, (2014).

16. RD 34.09.101-94. Typical instruction for electricity metering in its generation, transmission and distribution. M. ORGRES, S. (in Russian) (1995).

\section{Acknowledgments}

The reported study was funded by the Ministry of Education and Science of the Russian Federation under Federal Targeted Programme according to the agreement № 14.578.21.0226 (Project identifier: 